\title{
Kein gravierender Mangel mehr an Ärzten im Katastrophengebiet Aceh/Nordsumatra
}

In der Nr. 3 der Schweizerischen Ärztezeitung dieses Jahres ist ein von Herrn D. Seeger, PanEco, gezeichneter Artikel erschienen, in welchem von einem gravierenden Mangel an Ärzten im Katastrophengebiet von Nordsumatra die Rede ist. Es wird ein Arzt einer indonesischen Nicht-Regierungs-Umweltorganisation namens YEL zitiert, der davon spricht, dass dringend mindestens 500 Ärztinnen und Ärzte und 700 Pflegefachkräfte im Katastrophengebiet benötigt werden.

Dieser Aussage können die Unterzeichneten, die vom 3. bis 10. Januar persönlich in den Katastrophengebieten waren, davon drei Tage in Sumatra, heute nicht nachvollziehen. Auch die aktuellsten über ihre Informationskanäle einlaufenden Lageberichte und die direkten Kontakte mit Ärzten vor Ort, inklusive von PanEco und andern NGOs, lassen erkennen, dass punktuell zwar Bedarf an einzelnen Ärzte- und Pflegeteams besteht, vor allem auch zur Ablösung von heimkehrenden Teams. Diese Ablösungen lassen sich in der Regel von den betreffenden Organisationen problemlos selber rekrutieren.

Die Zahl der im Zusammenhang mit der Flutkatastrophe akut Behandlungsbedürftigen ist naturgemäss massiv zurückgegangen und es geht vor allem darum, die ärztliche Grundversor- gung und Spitalbehandlung der Überlebenden vor Ort und der obdachlos gewordenen und geflohenen Bevölkerung sicherzustellen und die Strukturen des Gesundheitswesens wieder aufzubauen. In einer zweiten Phase wird die Rehabilitation der vielen Opfer, die nur durch eine Amputation gerettet werden konnten, eine grosse Herausforderung darstellen.

Der eingangs zitierte Artikel hat eine ganze Reihe von Hilfsangeboten von Ärzten und Pflegeteams an die Unterzeichneten ausgelöst, die aber alle auf (möglicherweise) später vertröstet und damit oft enttäuscht werden müssen.

Zurzeit wird die Lage seitens der WHO, die für die internationale Koordination im Gesundheitswesen von der UNO mandatiert ist, laufend verfolgt, und die genauen Bedürfnisse werden entsprechend bekanntgegeben und durch die Humanitäre Hilfe weitergeleitet.

Toni Frisch, Delegierter für Humanitäre Hilfe

Daniel Biedermann, Direktor Schweizerisches Rotes Kreuz

Gianpiero A. Lupi, Chef Sanität/Oberfeldarzt der Armee 